\title{
Zur kommunikativen Bedeutung des Wortes «müssen» in der Psychotherapie: Die pathischen Kategorien
}

\author{
Manfred M. Fichter \\ Medizinisch-Psychosomatische Klinik Roseneck, Prien, und Klinik für Psychiatrie und Psychotherapie der LMU, München, Deutschland
}

In der klinischen Praxis werden wir immer wieder mit Patienten konfrontiert, die häufig das Wort müssen verwenden. Der Depressive sagt zum Beispiel: «Ich muss bald wieder gesund werden, damit ich wieder arbeiten kann» oder «Ich muss diese quälenden negativen Gedanken loswerden, oder ich bereite dem Ganzen ein Ende». Depression heißt zu Deutsch «niedergedrückt sein». Der Depressive macht sich selbst Druck. Es ist, als würde er sich mit jedem «Ich muss es, aber schaff' es nicht, weil ich nicht kann» resignativen Druck machen. Ähnlich macht sich der Patient mit chronischen Schmerzen oder Tinnitus unnötigen Druck, wenn er sich sagt «Der Schmerz/ Tinnitus muss aufhören». Wer sich auf diese Weise Druck macht, erhöht die innere und muskuläre Spannung mit dem Erfolg, dass sie nicht weggeht, sondern möglicherweise sogar stärker wird. Loslassen und ablenken wären hier hilfreicher. Das «Ich muss» verstärkt das Leiden.

Wir Therapeuten sind geneigt, dem Patienten vom Müssen zum Wollen zu verhelfen. Wir versuchen ihm Zuversicht zu geben, dass er es schaffen kann, dass er durchhalten soll, dass er sich seiner Ressourcen besinnen und sich erinnern soll, was er alles kann und früher geschafft hat. Und wir versuchen ihn dahin zu bringen, eine eigene Entscheidung zu fällen: «Ich treffe die freie Entscheidung, diese Expositionsübung heute Morgen zu machen. Ich tue dies, auch wenn ich Angst davor habe. Ich tue es, weil mein Verstand mir sagt, dass es besser sei, auf die Angst zuzugehen. Ich mache diese Übungen, damit ich seelisch und körperlich erfahre, dass sie langfristig das Beste für mich sind.» Zwischen dem Müssen und dem Wollen steht die Entscheidung des Patienten. Durch sie wird aus dem «Ich muss» ein «Ich will».

Dokumentarfilme über deutsche und österreichische Soldaten, die am Ende des Ersten Weltkrieges aus den hart umkämpften Südtiroler Bergen nach Süden in italienische Täler in Kriegsgefangenschaft geführt wurden, zeigen bei diesen einen sehr niedergeschlagenen Gesichtsausdruck. Bilder von
Touristen, die denselben Weg gehen, zeigen lachende Gesichter. Die einen mussten, die anderen durften und wollten diesen Weg gehen.

Manche Menschen, die sich mit den Worten «Ich muss» unter Druck setzen und den Erwartungen der Familie gehorchen, leisten auf dieses Weise Großes (Kennedy, Mann usw.). Andere maximieren den Druck, indem sie sich nicht nur sagen: «Ich muss diese Prüfung bestehen», sondern «Wenn ich diese Prüfung nicht mit einer Eins abschließe, bin ich ein Versager» (dichotomes Denken). Der selbstgemachte Druck teilt dann die, die sich damit «Rückenwind» verschaffen, von denen, die verzagen oder bei dem Übermaß an Druck versagen. Jene, die verzagten und versagten, sehen wir unter unseren Patienten; jene, die den extremen Druck aushielten und erfolgreich waren, sehen wir unter anderem in den Medien und bewundern ihren Erfolg. Dies mag erklären, warum sich das «dichotome Denken» im Laufe der Evolution des Menschen nicht «ausgemendelt» hat, sondern über die Generationen weitergegeben wurde. «Wat mut, dat mut», sagt eine plattdeutsche Volksweisheit.

Die genannten Beispiele führen uns in die gedankliche Welt Viktor von Weizsäckers (1886-1957) ein, der im Rahmen einer medizinischen Anthropologie und Krankheitslehre fünf pathische Kategorien - Kategorien des Leidens - beschrieb, indem er aus medizin-philosophischer Sicht Bedeutung und Kontext von fünf Verben erörterte, die er auch als «pathisches Pentagramm» bezeichnete [von Weizsäcker, 2005]: Müssen, Dürfen, Wollen, Sollen, Können. Der gefangene Soldat will nach Hause, aber er darf nicht und somit kann er auch nicht. Der Depressive will heraus aus seinem Jammertal, doch kann er den Weg nicht finden. Da Therapeuten häufig mit diesen fünf Kategorien des Leidens konfrontiert sind, ist es hilfreich, sich den Bedeutungsgehalt dieser Worte vor Augen zu führen. Im Folgenden gebe ich einige der Reflexionen dazu von Viktor von Weizsäcker wieder:

\begin{tabular}{ll}
\hline KARGER & @ 2007 S. Karger GmbH, Freiburg \\
Fax +497614520714 & Accessible online at: \\
$\begin{array}{l}\text { E-mail Information@Karger.de } \\
\text { www.karger.com }\end{array}$ & www.karger.com/ver
\end{tabular}


Vom Müssen schreibt von Weizsäcker, es sei der «Karfreitag des menschlichen Daseins (...) Das Müssen ist viel zu schlimm, als dass man es verdrängen dürfte; aber es ist nicht so schlimm, dass man es als Mittelpunkt auszeichnen (...) dürfte. Im Müssen ist der Tod, die Notwendigkeit, die Macht und der Zwang enthalten. (...) Das Müssen ist weder gut noch böse, sondern es ist gut und böse». Das Müssen steht in engem Zusammenhang mit den anderen vier pathischen Kathegorien. «Eine Person sagt 〈Ich muss nur das, was ich will〉. Eine andere sagt 〈Ich muss alles, was ich nicht will〉. Ein Kranker sagt 〈Ich muss vor allem gesund werden〉. Und ein Vierter sagt «Ich muss ein körperliches Bedürfnis verrichten〉. (...) Wer etwas muss, der seufzt. Das ist so sehr der Fall, dass, wer seufzt, etwas zu müssen scheint» [S. 79]. Von Weizsäcker sah auch die enge Verbindung zwischen Müssen und Wollen: «Wenn jemand z.B. sagt, 〈Ich muss〉, argwöhnen wir, er hätte besser gesagt 〈Ich will〉 usw. Dieses Transvestitentum ist am häufigsten dort anzutreffen, wo jemand sagt, 〈Ich muss das tun> und wir schon wissen, dass sein eigentliches Müssen sich nicht auf das bezieht, was er tun zu müssen vorgibt, sondern auf etwas ganz anderes, z.B. auf den Befehl, dem er folgen muss, und auf den, der den Befehl gab» [S. 80]. Er spricht von der Selbstverborgenheit des Müssens und zitiert aus Lessings «Nathan der Weise»: «Kein Mensch muss müssen». Er sieht im Müssen eine argwöhnisch machende Kategorie. Über den Zusammenhang von Müssen und Dürfen schreibt er: «Einen Schritt weiter kommt man mit der Einsicht, dass die Brunnenvergiftung nach menschlichem Ermessen nicht in der Quelle, sondern bereits entfernt nachweisbar ist, also dort, wo das Dürfen bereits ins Müssen degeneriert ist. Und an dieser Stelle taucht auch die Verneinung auf. Wer sagt, er müsse, der sagt auch, er könne nicht anders, er dürfe nicht anders; schließlich redet er sich und uns unter Umständen auch ein, er solle nicht und wolle nicht anders. Das Müssen ist nicht mehr so rein wie das Dürfen, es ist angefärbt von anderen pathischen Kategorien, und es ist repulsiv, es ist negierend gegen das andere. Die kategoriale Unfreiheit des Müssens steckt also auch in der Negation, und damit bekommen unsere Hauptbeispiele: der Tod, die Notwendigkeit, der Zwang und die Macht eine negative Färbung» [S. 83f].

Das Dürfen ist der «Ostermorgen des menschlichen Daseins». Seit der Aufklärung werden das Dürfen und Nichtdürfen sehr im gedanklichen Zusammenhang mit Freiheit gesehen. Von Weizsäcker sieht das normative Merkmal des Dürfens. Grenzensetzung und Grenzenerweiterung beeinflussen das Dürfen. «Das Dürfen begegnet uns überall gleichsam angefärbt von den anderen pathischen Kategorien, also vom Müssen, Sollen, Wollen und Können.» Dies zeigt sich in den Formulierungen: «Ich darf, was verboten ist» oder «Ich darf nichts und alles» oder «Ich darf immer tun, was ich mag» oder «Ich darf essen». Die Erfahrung lehrt, «dass ein Mensch eine üble Gewohnheit oder eine Sucht nur dann ablegt, wenn er muss. Das, was dann eintritt, ist also wieder ein Zwang, in dem er jetzt frei von dem ist, was er zuvor unter Zwang tat. Er darf also etwas lassen, wenn er es lassen muss» [S. 77].

Die Bedeutung des Wollens hat in der Neuzeit einen hohen und positiven Rang erhalten. In der Neuzeit entwickelte sich der Triumph des Willens. Aber man kann das Gute, jedoch auch das Böse wollen. Gewollt werden kann nur etwas, was nicht bereits ist oder vorliegt. Darin liegt der Leidensaspekt (Pathos) des Wollens; das Leiden, das entsteht, wenn wir etwas wollen, aber nicht dürfen oder können oder sollen. «Jeder Willensakt lässt Freiheit verschwinden und Notwendigkeit entstehen» [S. 88].

Das Sollen steht in enger Verbindung mit Gesetzen, Geboten, Gewissen, Moral und Ethik. Bei der Frage «Warum soll die Zofe das Kleid der Herrin eigentlich nicht tragen?» geht es um Besitzrecht. Damit steht das Sollen hier in enger Beziehung mit dem Dürfen. Die 10 Gebote listen auf, was wir nicht sollen: «Du sollst nicht töten». Treffender wäre es zu sagen: «Du darfst nicht töten». Der Begriff des Sollens steht auch in enger Beziehung zum Begriff des Widerstandes. Da gibt es den Patienten, der sich aus Eigensinn nicht operieren lassen will, den Raucher, der trotz aller Evidenzen gegen das Rauchen damit nicht aufhören will, und die Ungeduld des Patienten, der - obwohl schwer krank - nicht im Bett bleiben will. Im Gesundheitsbereich hat das Sollen ein besonderes Gewicht dadurch, dass Gesundheit der Sollzustand ist und Krankheit wie dessen Gegenteil dasteht.

Das Können ist die fünfte Kategorie des Leidens. Der Resignierte fragt sich, ob er denn jemals kann, was er will. Das Leiden liegt hier also im Nichtkönnen. Wenn der Kranke sagt «Ich kann nicht», bringt er ein Leiden zum Ausdruck. Anders wäre es, wenn er sagen würde: «Ich könnte, aber ich will nicht» oder «Dieses Mögliche ist ein Unmögliches für mich». Wie bei allen Kategorien des Leidens im Sinne von von Weizsäcker gibt es auch beim Können eine zeitliche Achse («Ich konnte es früher», «ich kann es jetzt nicht», «ich werde es in Zukunft nicht können») und eine Beschränkung des Raumes («Ich kann es an diesem Ort, aber nicht an jenem»).

Zurück zu unseren Patienten und dem klinischen Alltag. Müssen, Dürfen, Wollen, Sollen und Können sind wichtige Begriffe zum Verstehen des persönlichen Leidens. $\mathrm{Zu}$ jedem dieser fünf Worte gibt es auch einen Konjunktiv (ich dürfte, ich müsste, ich wollte, ich sollte, ich könnte), der die jeweilige Aussage abschwächt. Der Satz «Ich könnte heute mit dem Rauchen aufhören, aber spätestens morgen würde ich wieder anfangen», zeigt die Verniedlichung durch den Konjunktiv. Ist das Wortklauberei? Keineswegs. Es geht um aktives Zuhören in der Psychotherapie, um die Wahrnehmung von Nuancen, um Verhalten (in diesem Fall die Wortwahl des Patienten) und um Schärfung unserer psychotherapeutischen Sinne, diese Nuancierungen des Leidens wahrzunehmen. Wie können wir im Therapieprozess die Perspektive «Ich muss» (= leiden) zum «Ich kann» verändern? Wie können wir diese Metamorphose beim Patienten induzieren? Im Altertum waren es die Götter, die z.B. über den Ausgang einer Schlacht entschie- 
den und sich dabei persönlich und auch gegeneinander engagierten. Die Götter straften Erysichton, der den der Fruchtbarkeitsgöttin Ceres geweihten Baum fällte, mit einer absoluten Fresssucht: Er musste Hab und Gut versilbern, um genug Essen zu haben, und musste zur Strafe sogar sich selbst verzehren. Er konnte seiner Strafe nicht entrinnen. Die Verwandlung lag in der Strafe.

In unserer gegenwärtigen aufgeklärten Welt kann die Verwandlung auch durch Therapeuten bzw. durch den Patienten selbst erfolgen. Ein berühmtes Beispiel ist die ZaunstreichEpisode in Mark Twains «Tom Sawyer»: Toms Tante Polly hatte ihm als Strafe für einen Streich aufgetragen, dass er den Zaun streichen muss. Auf den ersten Blick sah es so aus, als sei dies als Strafe unabwendbar. Allerdings erkannte Tom, dass dieses «muss» nicht unveränderlich war. Als seine Freunde auf dem Weg zum Baden an diesem schönen Tag vorbeizogen, vermittelte er diesen (sie waren neugierig, was er da machte) eine andere Sichtweise: Zaunstreichen war etwas ganz Besonderes, was jetzt nur er durfte. Schließlich ließ er auf deren Drängen zu, dass seine Freunde auch den Pinsel in die Hand nahmen. Dafür, dass sie auch mal streichen durften, mussten sie aber mit ihren kleinen Schätzen (Murmeln, Zinnsoldat, zwei Kaulquappen usw.) bezahlen. Mark Twain kommentiert dies mit der Erkenntnis, «dass Arbeit das ist, was man tun muss und Spiel das ist, was man freiwillig tut» (darf). Sehr schnell war der Zaun gestrichen und jeder war zufrieden. Tom, weil er die Strafarbeit so elegant und schnell erledigt hatte, seine Freunde, die - nicht wissend, dass es Toms Strafarbeit war - den Zaun streichen durften, und Tante Polly, als sie sah, dass Tom seine Strafe brav erledigt hatte. Die Verwandlung (Metamorphose) fand im Kopf von Tom Sawyer statt. Er erkannte die Möglichkeit, das Müssen in ein Dürfen zu verwandeln. Er bediente sich der Konzepte der kognitiven Verhaltenstherapie lange, bevor es sie gab.

Karl Valentin (1882-1948) lebte etwa in derselben Zeit wie Viktor von Weizsäcker, und es war ihm vergönnt, die pathischen Kategorien komisch, witzig und verkürzt auf den Punkt zu bringen: «Mögen hätt' ich schon wollen, aber dürfen hab' ich mich nicht getraut!» [http://de.wikiquote.org/wiki/Karl_Valentin]

Nun zum eigentlichen Inhalt dieser Ausgabe von VerHALTENSTHERAPIE: Das vorliegende Heft enthält die Darstellung von fünf empirischen Studien sowie ein Konzeptpapier zur psychotherapeutischen Kompetenz. Jänsch et al. [2007] untersuchten Zwangspatienten mit frühem bzw. spätem Krankheitsbeginn. Im Zeitquerschnitt hatten Zwangskranke mit frühem Krankheitsbeginn vergleichsweise mehr und ausgeprägtere Zwangssymptome. Feldmann et al. [2007] untersuchten in einer randomisierten Studie die Wirksamkeit der Lektüre eines Elternratgebers (Triple P). Diese Bibliotherapie, unterstützt durch sieben Telefonate mit einem Experten, führte zu einer signifikanten Besserung kindlicher Symptome und Verhaltensweisen sowie positiven Änderungen im elterlichen Erziehungsverhalten. Interessant und praktisch relevant sind auch die Ergebnisse der empirischen Untersuchung von Crössmann et al. [2007]. Sie zeigten, dass eine detaillierte audiovisuelle Information vor eingreifenden diagnostischen Maßnahmen (Koronarangiographie) die Angst vor dem Eingriff bedeutsam vermindert. R. Böse [2007] untersuchte die positiven Auswirkungen eines Behandlungsbausteins, der speziell auf Körperschemastörungen fokussiert, für Patientinnen mit Anorexia nervosa. Falge-Kern et al. [2007] berichten über Ergebnisse einer quasi-experimentellen Untersuchung; stationäre PatientInnen mit einer Persönlichkeitsstörung nahmen in der Experimentalgruppe zusätzlich an einer psychoedukativen, kompetenz- und ressourcenorientierten Gruppenpsychotherapie teil. Diese erwies sich (im Vergleich zu Patienten, die diese Zusatzbehandlung nicht erhielten) als effektiv und sinnvoll. Linden et al. [2007] beschreiben ein mehrdimensionales Modell für psychotherapeutische Kompetenzen. Die sechs in diesem Modell dargestellten Dimensionen (z.B. Beziehungsmerkmale, Basiskompetenzen) werden als operationalisierbar, beobachtbar, bewertbar, lehrbar und erlernbar beschrieben. Da das Modell schulübergreifend ist, kann es auch zur Analyse von Gemeinsamkeiten und Unterschieden verschiedenartiger therapeutischer Ansätze verwendet werden. Diese Arbeiten müssen Sie unbedingt lesen.

\section{Literatur}

Böse R: Ernährungsmanagement versus Body-ImageTherapie bei Anorexia nervosa. Verhaltenstherapie DOI: $10.1159 / 000099305$.

Crössmann A, Voelker W, Pauli P, Bär S, Borst R, Spindler M: Angstreduktion durch audio-visuelle Information vor der Koronarangiographie. Verhaltenstherapie DOI: $10.1159 / 000099637$.

Falge-Kern A, Schulz H, Fricke S: Ein Gruppentherapieprogramm bei Persönlichkeitsstörungen und unflexiblen Persönlichkeitsstilen: Eine quasiexperimentelle Evaluation. Verhaltenstherapie DOI: 10.1159/000099657.
Feldmann M, Heinrichs N, Hahlweg K, Bertram H: Bibliotherapie zur Verbesserung elterlicher Erziehungskompetenz: Eine randomisierte Evaluationsstudie. Verhaltenstherapie DOI: 10.1159/000099629. Jänsch P, Zaudig M, Röper G, Hauke W, Piesbergen C, Butollo W: Der frühe Beginn der Zwangsstörung: Einfluss auf Symptomatik und Schweregrad. Verhaltenstherapie DOI: $10.1159 / 000097878$.

Linden M, Müller C, Milew D: Das Mehrebenen-Modell psychotherapeutischer Kompetenz. Verhaltenstherapie DOI: $10.1159 / 000098158$.
Valentin K: Texte von und über Karl Valentin; in Bachmaier H (Hrsg): Kurzer Rede langer Sinn. München, Piper, 1990. Quelle: http://de.wikiquote.org/wiki/ Karl_Valentin.

von Weizsäcker V: Pathosophie; in Achilles P, Janz D, Schrenk M, von Weizsäcker CF (Hrsg): Gesammelte Schriften. Frankfurt/M., Suhrkamp, 2005, Bd. 10, pp 67-105. 